Randomised control trial

\title{
People with unhealthy lifestyle behaviours benefit from remote coaching via mobile technology
}

\section{Faryle Nothwehr}

$10.1136 /$ eb-2012-100953

Correspondence to: Dr Faryle Nothwehr Community and Behavioral Health, University of lowa, 105 River Street, N424 CPHB, lowa City, IA52242,USA; faryle-nothwehr@uiowa.edu

Commentary on: Spring B, Schneider K, McFadden HG, et al. Multiple behavior changes in diet and activity: a randomized controlled trial using mobile technology behavior changes in diet and activity. Arch Intern Med 2012;172:789-96.

\section{Implications for practice and research}

- The study confirms previous research findings suggesting that behavioural interventions that encourage goal setting and frequent self-monitoring can result in significant, positive behavioural change, even in a population with fairly entrenched behavioural patterns.

- Non-targeted behaviours may be affected through a process of complementary behavioural change.

- Additional research is needed to better understand whether and how changes in non-targeted behaviours occur in adults, as this could lead to more efficient interventions.

- Use of mobile technology to encourage self-monitoring has the potential to provide exceptionally rich data for researchers studying the behaviour change process. However, the relative advantage of such technology over low-technology self-monitoring methods in terms of participant behaviour change is not known.

\section{Context}

Clustering of unhealthy lifestyle behaviours has been well documented. Addressing multiple behavioural issues at the same time presents a challenge for both practitioners and the clients they serve. This four-group randomised trial sought to examine the comparative impact of interventions focused on a combination of one aspect of dietary behaviour (low-fat diet or fruit and vegetable intake) and one aspect of physical activity (sedentary screen time or moderate to vigorous physical activity). The investigators hypothesised that the combination of increasing intake of fruits and vegetables and decreasing sedentary screen time would maximise healthy change by fostering healthy substitution (of fruits/vegetables for fat and physical activity for sedentary leisure) and complementary behaviour change (decreased fat intake accompanying decreased sedentary leisure).

\section{Methods}

The study involved a multiracial sample of 200 adults aged 21-60 years, recruited through community advertisements in a Midwest, USA, urban setting. Eligible participants were deficient of benchmark levels on all four behaviours. Interventions were delivered through 3 weeks of personal, remote coaching and utilised a hand-held electronic device for real-time self-monitoring and feedback regarding the individual's two target behaviours. Participants received incentives for reaching behavioural goals during an initial period, then only for reporting behaviours. Behavioural 
and other measures were taken at baseline and 5 months postintervention. A composite behavioural improvement score was created to indicate each participant's change over time across all behaviours. Multivariate modeling was used to analyse the data.

\section{Findings}

As hypothesised, the group focused on increasing fruits and vegetables and reducing sedentary screen time increased their composite behavioural score more than the other groups after 1 week ( $p=0.02$ ), and this advantage continued through the follow-up phase. In addition, decreased sedentary screen time correlated positively with reduced fat intake (an untargeted behaviour) ( $\mathrm{r}=0.29$, $\mathrm{p}=0.04$ ). Correlations among all other behaviour change pairs were not significant.

\section{Commentary}

This study utilised a novel design and innovative use of technology in intervention delivery. The participant sample was diverse and the analysis was rigorous. The funding that participants focused on fruit and vegetable intake and reducing sedentary screen time demonstrated a higher composite behavioural score seems to make sense as these behaviours may be easier to perform than others.

Reduced screen time has been widely and publicly promoted with the hope that this could increase physical activity as well as reduce intake of unhealthy snacking in children. ${ }^{1}$ These behavioural associations have not been thoroughly examined in adults. In this study, reduced screen time seemed to be associated only with reduced fat intake and not increased physical activity. However, in an earlier methods paper, Spring et $a l^{2}$ note that participants were coached to replace screen time with other sedentary activities (eg, reading and conversation). This was necessary to maintain fidelity to the study design. In real-world settings, it is likely that participants would be advised to, and/or would naturally try to, replace some of the screen time with physical activity, especially if weight management was the goal. Interestingly, increased fruit and vegetable intake was not associated with reduced fat intake, though one cannot rule out the presence of some low-level substitution. More studies are needed to explore these behavioural issues in adults.

The study demonstrated the feasibility of using mobile technology to encourage self-monitoring and self-regulation. Because all experimental groups used the technology, it is not known whether such systems are significantly more effective in supporting behavioural change than low-tech methods. There may be a strong novelty factor to the technology that encourages use, but it remains to be seen whether this would continue for longer than 5 months. The technology does, however, provide research advantages over simpler selfmonitoring methods in terms of data richness and detail.

Competing interests None.

\section{References}

1. CDC. Strategies and Solutions. Centers for Disease Control and Prevention; http://www.cdc.gov/obesity/childhood/solutions. html (accessed 13 Aug 2012).

2. Spring B, Schneider K, McFadden HG, et al. Make Better Choices (MBC): study design of a randomized controlled trial testing optimal technology-supported change in multiple diet and physical activity risk behaviors. BMC Public Health 2010;10:586. 